\title{
Absenteísmo-doença segundo autorrelato de servidores públicos municipais em Belo Horizonte
}

\author{
Celeste de Souza Rodrigues \\ Rosiene Maria de Freitas ${ }^{* *}$ \\ Ada Ávila Assunção** \\ lara Barreto Bassi ${ }^{\star \star \star * *}$ \\ Adriane Mesquita de Medeiros
}

O objetivo do estudo é analisar o perfil de um conjunto de servidores públicos municipais que relataram, por ocasião de um inquérito, absenteísmo-doença nos últimos 12 meses. Em 2009 obteve-se uma amostra de 5.646 (14\%) do universo de 38.304 servidores municipais de Belo Horizonte. Um questionário foi disponibilizado em sítio da Internet, cuja entrada dependia do consentimento do respondente. Regressão de Poisson foi utilizada para determinar os fatores associados. A prevalência do absenteísmo-doença foi maior entre os servidores do sexo feminino (RP 1,12) e diminuiu com o aumento das faixas etárias: 30-39 anos (RP 0,79); $40-49$ anos (RP 0,72); e 50 anos ou mais (RP 0,68). Absenteísmo-doença mostrou-se associado às atividades ligadas aos grupos de fiscalização e vigilância (RP 1,46), serviços gerais (RP 1,22), informação, educação e cultura (RP 1,50) e saúde (RP 1,50). As comorbidades mais presentes foram LER/Dort (RP 1,19), lombalgia (RP 1,16), depressão/ ansiedade (RP 1,20) e percepção de cansaço mental (RP 1,12). 0 relato de uso de medicação para depressão/ansiedade se mostrou fator protetor (RP 0,82). Maior probabilidade de transtorno mental (RP 1,23) segundo o SRQ20, insatisfação com a capacidade de trabalho (RP 1,08), condições razoáveis (RP 1,21) e condições precárias de trabalho (RP 1,19) foram associadas ao desfecho. Programas de promoção da saúde no trabalho visando a prevenção de comorbidades musculoesqueléticas e queixas psicossociais atenuariam as situações que provavelmente estão na origem do absenteísmo-doença.

Palavras-chave: Absenteísmo. Funcionários públicos. Doença. Condições de trabalho. Fatores psicossociais.

\footnotetext{
*Prefeitura de Belo Horizonte-MG, Brasil (celes@pbh.gov.br).

** Programa de Pós-Graduação em Ciência Política da Universidade Federal de Minas Gerais - UFMG, Belo Horizonte-MG, Brasil (rosiene@pbh.gov.br).

${ }^{* \star \star}$ Programa de Pós-Graduação em Saúde Pública da Universidade Federal de Minas Gerais - UFMG, Belo Horizonte-MG, Brasil (adavila@medicina.ufmg.br).

**** Programa de Pós-Graduação em Saúde Pública da Universidade Federal de Minas Gerais - UFMG, Belo Horizont-MG, Brasil (iara.bassi@hotmail.com).

***** Programa de Pós-Graduação em Saúde Pública da Universidade Federal de Minas Gerais - UFMG, Belo Horizonte-MG, Brasil (adrianemmedeiros@hotmail.com).
} 


\section{Introdução}

Absenteísmo, absentismo ou ausentismo corresponde à falta de assiduidade ao trabalho. Embora autores tenham sugerido nuanças nas definições, os termos são equivalentes para designar a ausência ao trabalho pelo empregado nas ocasiões em que seria esperada sua presença (EHRENBERG et al., 1989; ALVES; CERVINHO; GODOY, 2002). Absenteísmo refere-se ao período ininterrupto de falta ao trabalho, contado desde seu começo, independentemente de sua duração (EUROPEAN FOUNDATION, 1997).

O absenteísmo pode ser atribuído tanto a problemas de saúde como a causas diversas. Em sua origem costumam figurar as seguintes condições: doenças declaradas; doenças não declaradas; razões de caráter familiar; atrasos involuntários; faltas por dificuldades com transporte; perda de motivação; e debilidades da cultura organizacional (CHIAVENATO, 1981; MUNCH-HANSEN et al., 2008).

Absenteísmo-doença decorrente tanto de doença quanto de lesão acidental é aceito tanto pelo empregador quanto pela Previdência Social. Em 2005, no Brasil, mais de um bilhão de reais foram aplicados em benefícios auxílio-doença previdenciário, que cobrem os afastamentos do trabalho devido a uma morbidade declarada. No serviço público federal, as aposentadorias precoces e os afastamentos, naquele ano, custaram 300 milhões de reais para a União (CUNHA et al., 2009).

A ausência ao trabalho motivada por episódio de doença assume relevância na esfera do emprego público devido ao volume de licenças médicas e de dias não trabalhados no setor. Adicionalmente, o evento é mais frequente entre trabalhadores que lidam com atendimento ao público, particularmente entre aqueles que prestam cuidados a doentes. Em tais setores, as taxas de absenteísmo-doença podem variar de 15,9\% (SALA et al., 2009) a 60\% (SILVA; MARZIALE, 2000). Em um grupo de trabalhadores da enfermagem, apenas $13 \%$ foram assíduos no período de um ano e $87 \%$ apresentaram uma ou mais faltas, das quais $75 \%$ foram justificadas por licenças-médicas (SILVA; MARZIALE, 2000).

Recentemente, evidências justificam as abordagens que consideram o absenteísmodoença um indicador relevante para a saúde pública, porque, entre outros fatores, o risco de aposentadoria precoce e de mortalidade é elevado entre homens e mulheres com mais de 15 dias de licença médica por ano (ROELEN et al., 2010). Sabe-se também que o absenteísmodoença de longa duração está associado a uma pior avaliação da própria saúde (ERIKSSON et al., 2008).

Apesar de tais evidências, principalmente no setor público, o fenômeno tem sido visto mais como uma questão socioeconômica e política do que um problema de saúde pública. Saliente-se que as preocupações dos gestores estão motivadas pelos gastos financeiros e diminuição da produtividade, razões comumente evocadas nas instituições (BOWERS, 2001).

Entretanto, o fenômeno mundial de caráter multidimensional pode ser o resultado tanto de queixas inespecíficas quanto de doenças declaradas, sendo que a ocorrência e evolução são influenciadas por fatores sociodemográficos, comportamentais, ocupacionais, etc. (DUIJTS 
et al., 2007). Quanto às morbidades, são mais frequentes os afastamentos por doenças do aparelho respiratório, doenças do sistema musculoesquelético e transtornos mentais (REIS et al., 2003; ERIKSSON et al., 2008).

No Brasil, autores já analisaram trabalhadores no setor de serviços, independentemente do caráter público ou privado, por exemplo, bancários, servidores universitários e trabalhadores de hospitais. Escassos são os estudos que abordaram o absenteísmo-doença em servidores públicos de uma única instituição, mas inseridos em diferentes ocupações e segundo os relatos dos próprios sujeitos. O presente trabalho tem o objetivo de analisar fatores associados ao absenteísmo-doença autorrelatado por servidores públicos municipais ativos que declararam, por ocasião de um inquérito, absenteísmo-doença nos últimos 12 meses.

\section{Métodos}

Foi realizado um inquérito epidemiológico, entre setembro e dezembro de 2009, cuja população-alvo constituiu-se de 38.304 servidores municipais de Belo Horizonte (Tabela 1). Foi feita ampla divulgação junto às gerências e trabalhadores, por meio de reuniões com o corpo gestor em todos os órgãos da Prefeitura, chamadas na Internet e Intranet (http:// portalpbh.pbh.gov.br), mensagens em contracheques, entre outros, com a finalidade de esclarecer os objetivos e a importância da participação. Esta divulgação buscou adesão da totalidade dos elegíveis. Os sindicatos representativos dos servidores e empregados públicos municipais foram acionados e sensibilizados.

Balanços sistematizados do número de questionários preenchidos foram elaborados semanalmente pela empresa de informática do município, permitindo acompanhamento e identificação da adesão de acordo com os setores das administrações direta e indireta. Desse modo, a coordenação reforçou a atuação da equipe de comunicação da pesquisa em áreas de fraca participação. Ao final, obteve-se uma amostra não probabilística de 5.646 (14\%).

As respostas ao questionário disponibilizado em sítio da Internet foram viabilizadas exclusivamente após o consentimento em participar. O termo de consentimento livre e esclarecido foi aprovado pelo Comitê de Ética em Pesquisa da Prefeitura de Belo Horizonte (Parecer n. 0054.0.410.000.09a).

A Tabela 1 apresenta as porcentagens de respondentes e do universo elegível de servidores, segundo faixa etária, sexo e escolaridade. Observa-se que ambas as populações são similares no que se refere à distribuição por sexo e faixa etária. Contrastando com o universo, entre os respondentes predominou nível superior ou pós-graduação.

A variável resposta foi medida por meio da questão "Você teve alguma licença/atestado médico por motivo de doença nos últimos 12 meses?”, com as seguintes alternativas: Não; Sim, 1 ou 2 licenças/atestados médicos; Sim, 3 licenças/atestados médicos; Sim, 4 ou mais licenças/atestados médicos. As respostas foram dicotomizadas em duas categorias: Não; Sim (1 ou mais licenças/atestados médicos). 
TABELA 1

Distribuição dos servidores e empregados públicos municipais das administrações direta e indireta e daqueles que responderam ao questionário da pesquisa, segundo características estudadas Município de Belo Horizonte - 2009

\begin{tabular}{|c|c|c|c|c|}
\hline \multirow{2}{*}{$\begin{array}{c}\text { Características } \\
\text { estudadas }\end{array}$} & \multicolumn{2}{|c|}{ Total de servidores } & \multicolumn{2}{|c|}{ Respondentes da pesquisa } \\
\hline & N. abs. & $\%$ & N. abs. & $\%$ \\
\hline Total & 38.304 & 100,0 & 5.646 & 100,0 \\
\hline Homens & 11.453 & 29,9 & 1.729 & 30,6 \\
\hline Mulheres & 26.851 & 70,1 & 3.632 & 64,3 \\
\hline Missing & - & - & 285 & 5,0 \\
\hline \multicolumn{5}{|l|}{ Faixa etária } \\
\hline 18 a 19 anos & 38 & 0,1 & 5 & 0,1 \\
\hline 20 a 29 anos & 5.209 & 13,6 & 855 & 15,1 \\
\hline 30 a 39 anos & 9.768 & 25,5 & 1.367 & 24,2 \\
\hline 40 a 49 anos & 13.445 & 35,1 & 1.848 & 32,7 \\
\hline 50 a 59 anos & 7.929 & 20,7 & 1.023 & 18,1 \\
\hline 60 anos e mais & 1.915 & 5,0 & 198 & 3,5 \\
\hline Missing & - & - & 350 & 6,2 \\
\hline \multicolumn{5}{|l|}{ Escolaridade } \\
\hline Analfabeto & 84 & 0,2 & 26 & 0,5 \\
\hline Ensino fundamental & 4.765 & 12,4 & 448 & 7,9 \\
\hline Ensino médio & 13.039 & 34,0 & 1.336 & 23,7 \\
\hline Superior & 15.433 & 40,3 & 1.713 & 30,3 \\
\hline Pós-Graduação & 4.983 & 13,0 & 1.831 & 32,4 \\
\hline Missing & - & - & 292 & 5,2 \\
\hline
\end{tabular}

Fonte: Diagnóstico de Saúde do Servidor e do Empregado Público da Prefeitura Municipal de Belo Horizonte.

Reconhecendo o caráter multidimensional do absenteísmo-doença na presente investigação, foram incluídos os fatores individuais (características demográficas e funcionais; e hábitos de vida e situação de saúde) e os fatores de contexto (qualidade de vida e qualidade do trabalho) que embasaram a construção dos eixos, os quais, por sua vez, orientaram a construção do instrumento de coleta de dados. Sob esse prisma, as variáveis explicativas foram agrupadas em domínios específicos:

- características demográficas e funcionais - sexo, idade, situação conjugal, gravidez atual, filhos, escolaridade, tempo que trabalha na unidade, grupos ocupacionais;

- hábitos de vida e saúde - atividade de lazer, atividade física, índice de massa corporal (IMC), tabagismo, diagnóstico médico de LER/Dort, diagnóstico médico de lombalgia, diagnóstico médico de obesidade, diagnóstico médico de depressão/ansiedade, diagnóstico médico de distúrbio do sono, percepção de cansaço mental, percepção de irritação, percepção de nervosismo, medicamento para depressão/ansiedade;

- qualidade de vida e trabalho - transtorno mental comum (SRQ-20), demanda psicossocial do trabalho (JSS), suporte social no trabalho, qualidade de vida, capacidade de trabalho, demanda física no trabalho, condições de trabalho.

As 384 ocupações ou funções exercidas na Prefeitura de Belo Horizonte foram agrupadas segundo critérios que classificam o ambiente organizacional, o qual designa a área específica de atuação do servidor, integrada por atividades afins ou complementares e organizada a 
partir das necessidades institucionais, orientando a política de desenvolvimento de pessoal (Lei n. 11.091, de 12 de janeiro de 2005). Para fins das análises, seis categorias foram elaboradas, conforme explicita o Quadro 1.

\section{QUADRO 1}

Ocupação ou função exercida na Prefeitura de Belo Horizonte, segundo categorias ocupacionais

\begin{tabular}{|ll|}
\hline Categorias ocupacionais & \multicolumn{1}{c|}{ Ocupação ou função exercida na Prefeitura Municipal de Belo Horizonte } \\
\hline $\begin{array}{l}\text { Administrativo, contábil, } \\
\text { financeiro, jurídico e } \\
\text { planejamento }\end{array}$ & $\begin{array}{l}\text { Analistas administrativos de nível superior, assessores, gerentes, chefes, diretores, } \\
\text { técnico de nível superior administração, técnico de nível superior contabilidade, } \\
\text { técnico de nível superior direito, técnico de nível superior economia, secretários, } \\
\text { agentes, técnicos, assistentes e auxiliares administrativos, etc. }\end{array}$ \\
\hline Fiscalização e vigilância & $\begin{array}{l}\text { Auditores fiscais, sanitarista, secretário de junta fiscal, técnico de segurança do } \\
\text { trabalho, agente de combate de endemia, fiscal municipal de controle ambiental, } \\
\text { fiscal de limpeza urbana, etc. }\end{array}$ \\
\hline Serviços gerais & $\begin{array}{l}\text { Técnico em mecânica, técnico em manutenção de equipamentos, supervisor, } \\
\text { serralheiro, tratador de animais, auxiliar de serviços gerais, braçal, operador de } \\
\text { máquinas pesadas, etc. }\end{array}$ \\
\hline $\begin{array}{l}\text { Construção e exploração } \\
\text { de recursos naturais }\end{array}$ & $\begin{array}{l}\text { Engenheiros, arquitetos, assistente técnico projetista, desenhista, técnico em } \\
\text { edificações, geólogo, analista ambiental, técnico em agrimensura, geógrafo, } \\
\text { técnico de nível médio paisagismo, etc. }\end{array}$ \\
\hline $\begin{array}{l}\text { Informação, educação e } \\
\text { cultura }\end{array}$ & $\begin{array}{l}\text { Analista de informática, técnico em eletrônica, técnico em processamento de } \\
\text { dados, técnico nível superior jornalismo, técnico nível superior comunicação } \\
\text { social, analista de sistema, educador infantil, professor, auxiliar de escola, técnico } \\
\text { superior de educação, educador social, técnico de turismo, etc. }\end{array}$ \\
\hline Saúde & $\begin{array}{l}\text { Médico, psicólogo, fisioterapeuta, farmacêutico, nutricionista, biólogo, agente } \\
\text { de serviço de saúde, técnicos e auxiliares de enfermagem, técnico em nutrição, } \\
\text { técnico em radiologia, técnico de laboratório, etc. }\end{array}$ \\
\hline
\end{tabular}

Para constituir a variável demanda física no trabalho, obteve-se a soma de escores das respostas a seis perguntas do questionário referentes aos aspectos postura, esforço físico e pausa para desempenho das atividades ocupacionais. Tais perguntas foram baseadas no questionário francês de avaliação da percepção sobre o trabalho em um grupo de assalariados franceses (CAIL; APTEL; PICHENE, 1995). Não foi realizada a validação desse questionário no Brasil. Para cada uma das quatro respostas possíveis a cada pergunta, foi atribuída uma pontuação conforme a seguinte escala: 1 ponto = nunca; 2 pontos = raramente; 3 pontos = às vezes; e 4 pontos = sempre. Efetuou-se a soma dos escores do bloco, que variou de 6 a 24 pontos. Pela mediana, a variável demanda física foi dividida em alta e baixa. Os valores iguais ou abaixo do ponto de corte foram enquadrados como baixa demanda e, aqueles acima deste ponto, como alta demanda.

A variável condições de trabalho foi constituída pela agregação das respostas referentes aos aspectos ruído, mobiliário, ventilação, temperatura, iluminação e recursos técnicos utilizados no trabalho. Também atribuiu-se uma pontuação para cada uma das três possíveis respostas a cada uma das perguntas: 1 ponto = precária; 2 pontos = razoável; e 3 pontos = satisfatória. Após a soma dos escores, obtiveram-se quatro categorias para a condição de trabalho: precária (escores abaixo do $1^{\circ}$ quartil); razoável (escores entre o $1^{\circ}$ e $2^{\circ}$ quartil); satisfatória (escores entre o $2^{\circ}$ e $3^{\circ}$ quartil); e ótima (escores acima do $3^{\circ}$ quartil). 
O DCQ (Demand Control Questionnaire) avalia as dimensões demanda psicológica, controle sobre o trabalho e apoio social no trabalho, referentes às fontes de estresse no ambiente psicossocial do trabalho e desgaste resultante de sua interação. Estas dimensões foram avaliadas pela versão brasileira reduzida (17 questões) do questionário sueco Demand, Control e Support - DCSQ (THEORELL; KARASEK, 1996), que, por sua vez, foi baseado no Job Content Questionnaire - JCQ (KARASEK, 1979; ALVES et al., 2004; GRIEP et al., 2010). A demanda psicológica - avaliada por meio de perguntas sobre os aspectos quantitativos (quatro questões) e qualitativos (uma questão) do processo de trabalho - reflete quão duramente o indivíduo trabalha e refere-se à pressão temporal, ao esforço mental e ao estímulo necessário ao cumprimento da tarefa. Estão contemplados a necessidade de coordenação de cargas, os estressores provenientes de conflitos interpessoais e o medo de obsolescência e de perder o emprego. O controle sobre o trabalho é avaliado por questões relacionadas ao uso e desenvolvimento de habilidades (quatro questões), enquanto a autoridade para tomada de decisão e o suporte social são analisados, respectivamente, por duas e seis questões.

Para avaliar a saúde mental, elaborou-se uma variável denominada transtorno mental comum (TMC), a qual expressa respostas às 20 questões fechadas que compõem o Self Reporting Questionnaire (SRQ20). Este instrumento tem a finalidade de identificar casos suspeitos de TMC em populações, caracterizados por insônia, fadiga, irritabilidade, esquecimento, dificuldade de concentração e queixas somáticas. Para fins desta análise, considerou-se ponto de corte sete para afirmar suspeição de TMC (SANTOS et al., 2009).

Os procedimentos de análise incluíram descritiva dos dados por meio de medidas absolutas e porcentagens. Posteriormente, para a análise dos fatores associados ao absenteísmo, foi utilizada a regressão de Poisson, que permite verificar a magnitude da associação de cada fator por meio da razão de prevalência (RP) e a significância estatística pelo intervalo de $95 \%$ de confiança. Como a razão de prevalência não pode ser diretamente derivada das equações de regressão logística, optou-se por estimá-la por meio da regressão de Poisson. Usualmente, o método é utilizado para análise de coortes longitudinais em que a variável dependente é uma contagem do número de ocorrências de um evento ao longo do tempo. No caso de estudos transversais, em que não há seguimento da amostra, o tempo pode ser ajustado considerando-se o tempo sob risco para cada indivíduo igual a um. Essa medida permitirá uma estimativa da prevalência de ponto. Uma vez que a regressão de Poisson aplicada a estudos transversais pode gerar uma superestimativa das medidas de associação, empregou-se o método de variância robusta, que permite convergir os resultados a achados similares aos obtidos pelo método de Mantel-Haenszel (BARROS; HIRAKATA, 2003; COUTINHO et al., 2008).

Todos os fatores associados ao nível de $p \leq 0,20$ na análise univariada foram incluídos no modelo multivariado por meio de deleção sequencial de variáveis. O modelo final multivariado contemplou as variáveis associadas ao nível de $p \leq 0,05$. A análise utilizou o programa Stata, versão 10.0. (Stata Corp., College Station, Estados Unidos). 


\section{Resultados}

Na análise univariada, a prevalência do absenteísmo-doença foi maior entre os servidores do sexo feminino (RP 1,39) e diminuiu com o aumento das faixas etárias: $30-39$ anos (RP 0,93); 40-49 anos (RP 0,86); e 50 anos ou mais (RP 0,76). Quanto à escolaridade, as prevalências foram maiores entre os servidores com ensino médio, técnico ou superior incompleto (RP 1,25) e ensino superior completo ou pós-graduação (RP 1,25). Observouse maior prevalência entre aqueles que trabalhavam na unidade em período inferior a dez anos, em contraposição àqueles que relataram trabalhar na mesma unidade há mais de 21 anos (RP 0,82). As categorias ocupacionais com maior prevalência de absenteísmo foram: fiscalização e vigilância (RP 1,32); informação, educação e cultura (RP 1,69); e saúde (RP 1,56) (Tabela 2).

TABELA 2

Distribuição dos servidores e empregados públicos municipais das administrações direta e indireta que responderam ao questionário da pesquisa, por situação de absenteísmo-doença, e razão de prevalência univariada, segundo características demográficas e funcionais Município de Belo Horizonte - 2009

\begin{tabular}{|c|c|c|c|c|c|c|}
\hline \multirow{3}{*}{$\begin{array}{l}\text { Características } \\
\text { demográficas e } \\
\text { funcionais }\end{array}$} & \multicolumn{4}{|c|}{ Absenteísmo-doença } & \multirow{3}{*}{$\begin{array}{c}\text { Razão de } \\
\text { prevalência }\end{array}$} & \multirow{3}{*}{ IC (95\%) } \\
\hline & \multicolumn{2}{|c|}{ Não } & \multicolumn{2}{|c|}{ Sim } & & \\
\hline & N. abs. & $\%$ & N. abs. & $\%$ & & \\
\hline \multicolumn{7}{|l|}{ Sexo } \\
\hline Homens & 1.067 & 37,9 & 858 & 25,7 & 1 & \\
\hline Mulheres & 1.747 & 62,1 & 1.690 & 74,3 & 1,39 & $1,29-1,49^{\text {***}}$ \\
\hline \multicolumn{7}{|l|}{ Idade } \\
\hline Até 29 anos & 405 & 14,6 & 419 & 18,6 & 1 & \\
\hline De 30 a 39 anos & 690 & 24,8 & 619 & 27,5 & 0,93 & $0,85-1,02^{*}$ \\
\hline De 40 a 49 anos & 977 & 35,2 & 766 & 34,0 & 0,86 & $0,79-0,94^{\star \star \star}$ \\
\hline 50 anos ou mais & 707 & 25,4 & 447 & 19,9 & 0,76 & $0,69-0,85^{\star \star \star}$ \\
\hline \multicolumn{7}{|l|}{ Situação conjugal } \\
\hline Sem companheiro(a) & 1.219 & 43,3 & 1.031 & 45,4 & 1 & \\
\hline Com companheiro(a) & 1.593 & 56,7 & 1.241 & 54,6 & 0,96 & $0,90-1,02^{\star}$ \\
\hline \multicolumn{7}{|l|}{ Gravidez atual } \\
\hline Não & 1.720 & 98,6 & 1.655 & 98,0 & 1 & \\
\hline $\operatorname{Sim}$ & 25 & 1,4 & 33 & 2,0 & 1,16 & $0,92-1,46^{*}$ \\
\hline \multicolumn{7}{|l|}{ Filhos } \\
\hline Não possui filho & 1.032 & 36,6 & 873 & 38,3 & 1 & \\
\hline Possui 1 ou 2 filhos & 1.328 & 47,1 & 1.069 & 46,9 & 0,97 & $0,91-1,04$ \\
\hline Possui 3 ou mais filhos & 458 & 16,3 & 337 & 14,8 & 0,93 & $0,84-1,02^{*}$ \\
\hline \multicolumn{7}{|l|}{ Escolaridade } \\
\hline Ensino fundamental & 243 & 8,6 & 138 & 6,1 & 1 & \\
\hline $\begin{array}{l}\text { Ensino médio, técnico } \\
\text { ou superior incompleto }\end{array}$ & 725 & 25,8 & 599 & 26,4 & 1,25 & $1,08-1,45^{\star \star}$ \\
\hline $\begin{array}{l}\text { Ensino superior } \\
\text { completo ou pós- } \\
\text { graduação }\end{array}$ & 1.844 & 65,6 & 1.535 & 67,6 & 1,25 & $1,09-1,44^{\star \star \star}$ \\
\hline
\end{tabular}


(continuação)

\begin{tabular}{|c|c|c|c|c|c|c|}
\hline \multirow{3}{*}{$\begin{array}{c}\text { Características } \\
\text { demográficas e } \\
\text { funcionais }\end{array}$} & \multicolumn{4}{|c|}{ Absenteísmo-doença } & \multirow{3}{*}{$\begin{array}{c}\text { Razão de } \\
\text { prevalência }\end{array}$} & \multirow{3}{*}{ IC (95\%) } \\
\hline & \multicolumn{2}{|c|}{ Não } & \multicolumn{2}{|c|}{ Sim } & & \\
\hline & N. abs. & $\%$ & N. abs. & $\%$ & & \\
\hline \multicolumn{7}{|l|}{$\begin{array}{l}\text { Tempo que trabalha na } \\
\text { unidade }\end{array}$} \\
\hline Até 10 anos & 1.921 & 68,8 & 1.609 & 71,4 & 1 & \\
\hline De 11 a 20 anos & 463 & 16,6 & 400 & 17,8 & 1,02 & $0,94-1,10$ \\
\hline Mais de 21 anos & 409 & 14,6 & 243 & 10,8 & 0,82 & $0,74-0,91^{\star \star \star}$ \\
\hline \multicolumn{7}{|l|}{ Grupos ocupacionais } \\
\hline $\begin{array}{l}\text { Administrativo, } \\
\text { contábil, financeiro, } \\
\text { jurídico e planejamento }\end{array}$ & 1.053 & 39,2 & 533 & 24,8 & 1 & \\
\hline Fiscalização e vigilância & 152 & 5,7 & 122 & 5,7 & 1,32 & $1,14-1,54^{\star \star \star}$ \\
\hline Serviços gerais & 334 & 12,4 & 181 & 8,4 & 1,05 & $0,91-1,20$ \\
\hline $\begin{array}{l}\text { Construção e } \\
\text { exploração de recursos } \\
\text { naturais }\end{array}$ & 72 & 2,7 & 35 & 1,6 & 0,97 & $0,74-1,29$ \\
\hline $\begin{array}{l}\text { Informação, educação } \\
\text { e cultura }\end{array}$ & 447 & 16,7 & 583 & 27,2 & 1,69 & $1,54-1,83^{\star \star \star}$ \\
\hline Saúde & 625 & 23,3 & 693 & 32,3 & 1,56 & $1,44-1,71^{\star \star \star}$ \\
\hline
\end{tabular}

Fonte: Diagnóstico de Saúde do Servidor e do Empregado Público da Prefeitura Municipal de Belo Horizonte.

${ }^{*} p<0,20 ;{ }^{* *} p<0,01 ;{ }^{* * *} p<0,001$.

Em relação aos comportamentos daqueles que relataram absenteísmo, a prática de atividade física de uma a duas vezes por semana (RP 0,86) e três ou mais vezes por semana (RP 0,78) foi fator protetor, com um gradiente dose-resposta na associação. A prevalência de absenteísmo foi maior para os obesos (RP 1,11) e aqueles que informaram diagnósticos médicos confirmados para LER/Dort (RP 1,46), lombalgia (RP 1,46), sobrepeso ou obesidade (RP 1,25), depressão/ansiedade (RP 1,73) e distúrbio do sono (RP 1,51). De acordo com a autopercepção, houve maior prevalência entre os que relataram cansaço mental (RP 1,65), irritação (RP 1,54) e nervosismo (RP 1,52). O relato de uso de medicação para depressão/ansiedade se mostrou fator protetor (RP 0,59) (Tabela 3).

Maior probabilidade de TMC (RP 1,74) foi encontrada entre os respondentes que informaram absenteísmo. Sobre a demanda psicossocial do trabalho, os servidores com alto desgaste (RP 1,24) apresentaram maior prevalência de absenteísmo. Baixo desgaste (RP 0,81) e baixo suporte social (RP 0,72) foram fatores protetores (Tabela 4).

A prevalência de absenteísmo foi maior entre os servidores que avaliaram ruim a qualidade de vida (RP 1,44) e entre aqueles que se declararam insatisfeitos com a capacidade de trabalho (RP 1,33) e expostos a alta demanda física no trabalho (RP 1,33). A prevalência de absenteísmo aumenta à medida que as condições de trabalho são negativamente avaliadas: satisfatórias (RP 1,25); razoáveis (RP 1,55); e precárias (RP 1,65) (Tabela 4). 


\section{TABELA 3}

Distribuição dos servidores e empregados públicos municipais das administrações direta e indireta que responderam ao questionário da pesquisa, por situação de absenteísmo-doença, e razão de prevalência univariada, segundo características relacionadas aos hábitos de vida e saúde

Município de Belo Horizonte - 2009

\begin{tabular}{|c|c|c|c|c|c|c|}
\hline \multirow{3}{*}{$\begin{array}{l}\text { Características relacionadas } \\
\text { aos hábitos de vida e saúde }\end{array}$} & \multicolumn{4}{|c|}{ Absenteísmo-doença } & \multirow{3}{*}{$\begin{array}{c}\text { Razão de } \\
\text { prevalência }\end{array}$} & \multirow{3}{*}{ IC (95\%) } \\
\hline & \multicolumn{2}{|c|}{ Não } & \multicolumn{2}{|c|}{ Sim } & & \\
\hline & N. abs. & $\%$ & N. abs. & $\%$ & & \\
\hline \multicolumn{7}{|l|}{ Realiza atividade de lazer } \\
\hline $\operatorname{Sim}$ & 2.419 & 82,4 & 1.918 & 81,2 & 1 & \\
\hline Não & 516 & 17,6 & 445 & 18,8 & 1,05 & $0,97-1,13$ \\
\hline \multicolumn{7}{|l|}{ Realiza atividade física } \\
\hline Não & 1.324 & 45,1 & 1.280 & 54,1 & 1 & \\
\hline \multirow{2}{*}{$\begin{array}{l}\text { Sim, } 1 \text { a } 2 \text { vezes/semana } \\
\text { Sim, } 3 \text { ou mais vezes/ } \\
\text { semana }\end{array}$} & 821 & 28,0 & 599 & 25,3 & 0,86 & $0,80-0,92^{\star *}$ \\
\hline & 790 & 26,9 & 488 & 20,6 & 0,78 & $0,72-0,84^{\star \star}$ \\
\hline \multicolumn{7}{|l|}{$\mathrm{IMC}$} \\
\hline Baixo ou normal & 1.470 & 56,6 & 1.171 & 55,4 & 1 & \\
\hline Sobrepeso & 822 & 31,7 & 647 & 30,6 & 0,99 & $0,92-1,07$ \\
\hline Obesidade & 305 & 11,7 & 294 & 13,9 & 1,11 & $1,01-1,21^{*}$ \\
\hline \multicolumn{7}{|l|}{ Tabagismo } \\
\hline $\begin{array}{l}\text { Não (não fumante ou ex- } \\
\text { fumante) }\end{array}$ & 2.613 & 89,2 & 2.112 & 89,6 & 1 & \\
\hline Sim (fumante atual) & 316 & 10,8 & 245 & 10,4 & 0,98 & $0,88-1,08$ \\
\hline \multicolumn{7}{|l|}{$\begin{array}{l}\text { Possui diagnóstico médico } \\
\text { de LER/Dort }\end{array}$} \\
\hline Não & 2.753 & 93,1 & 2.045 & 85,9 & 1 & \\
\hline Sim & 240 & 6,9 & 336 & 14,1 & 1,46 & $1,36-1,57^{\star \star}$ \\
\hline \multicolumn{7}{|l|}{$\begin{array}{l}\text { Possui diagnóstico médico } \\
\text { de lombalgia }\end{array}$} \\
\hline Não & 1.922 & 64,9 & 1.142 & 47,9 & 1 & \\
\hline $\operatorname{sim}$ & 1.038 & 35,1 & 1.240 & 52,1 & 1,46 & $1,38-1,55^{\star \star}$ \\
\hline \multicolumn{7}{|l|}{$\begin{array}{l}\text { Possui diagnóstico médico } \\
\text { de obesidade }\end{array}$} \\
\hline Não & 2.499 & 84,6 & 1.857 & 78,1 & 1 & \\
\hline Sim & 456 & 15,4 & 522 & 21,9 & 1,25 & $1,17-1,34^{\star \star}$ \\
\hline \multicolumn{7}{|l|}{$\begin{array}{l}\text { Possui diagnóstico médico } \\
\text { de depressão/ansiedade }\end{array}$} \\
\hline Não & 2.553 & 86,4 & 1.586 & 66,8 & 1 & \\
\hline Sim & 403 & 13,6 & 788 & 33,2 & 1,73 & $1,63-1,83^{\star *}$ \\
\hline \multicolumn{7}{|l|}{$\begin{array}{l}\text { Possui diagnóstico médico } \\
\text { de distúrbio do sono }\end{array}$} \\
\hline Não & 2.424 & 82 & 1.586 & 66,6 & 1 & \\
\hline Sim & 532 & 18 & 795 & 33,4 & 1,51 & $1,43-1,61^{\star *}$ \\
\hline \multicolumn{7}{|l|}{ Percepção de cansaço mental } \\
\hline Não & 2.267 & 76,6 & 1.320 & 55,4 & 1 & \\
\hline Sim & 693 & 23,4 & 1.064 & 44,6 & 1,65 & $1,55-1,74^{\star \star}$ \\
\hline \multicolumn{7}{|l|}{ Percepção de irritação } \\
\hline Não & 2.474 & 83,6 & 1.627 & 68,2 & 1 & \\
\hline Sim & 485 & 16,4 & 757 & 31,8 & 1,54 & $1,45-1,68^{\star \star}$ \\
\hline \multicolumn{7}{|l|}{ Percepção de nervosismo } \\
\hline Não & 2.419 & 81,7 & 1.576 & 66,1 & 1 & \\
\hline Sim & 541 & 18,3 & 808 & 33,9 & 1,52 & $1,43-1,61^{\star *}$ \\
\hline \multicolumn{7}{|l|}{$\begin{array}{l}\text { Usa medicamento para } \\
\text { depressão/ansiedade }\end{array}$} \\
\hline Não & 2.633 & 89 & 1.718 & 72,2 & 1 & \\
\hline Sim & 326 & 11 & 663 & 27,8 & 0,59 & $0,56-0,62^{\star \star}$ \\
\hline
\end{tabular}

Fonte: Diagnóstico de Saúde do Servidor e do Empregado Público da Prefeitura Municipal de Belo Horizonte.

${ }^{\star} p<0,05 ;{ }^{*} p<0,001$. 


\section{TABELA 4}

Distribuição dos servidores e empregados públicos municipais das administrações direta e indireta que responderam ao questionário da pesquisa, por situação de absenteísmo-doença, e razão de prevalência univariada, segundo características relacionadas à qualidade de vida e ao trabalho

Município de Belo Horizonte - 2009

\begin{tabular}{|c|c|c|c|c|c|c|}
\hline \multirow{3}{*}{$\begin{array}{l}\text { Características relacionadas à } \\
\text { qualidade de vida e ao trabalho }\end{array}$} & \multicolumn{4}{|c|}{ Absenteísmo-doença } & \multirow{3}{*}{$\begin{array}{l}\text { Razão de } \\
\text { prevalência }\end{array}$} & \multirow{3}{*}{ IC (95\%) } \\
\hline & \multicolumn{2}{|c|}{ Não } & \multicolumn{2}{|c|}{ Sim } & & \\
\hline & N. abs. & $\%$ & N. abs. & $\%$ & & \\
\hline \multicolumn{7}{|l|}{ Transtorno mental comum (SRQ20) } \\
\hline $\begin{array}{l}\text { Menor probabilidade de transtorno } \\
\text { mental }\end{array}$ & 2.204 & 81,8 & 1.269 & 59,7 & 1 & \\
\hline $\begin{array}{l}\text { Maior probabilidade de transtorno } \\
\text { mental }\end{array}$ & 491 & 18,2 & 858 & 40,3 & 1,74 & $1,64-1,85^{\star}$ \\
\hline \multicolumn{7}{|l|}{ Demanda psicossocial do trabalho (JSS) } \\
\hline Trabalho ativo & 597 & 21,8 & 485 & 22,1 & 1 & \\
\hline Alto desgaste & 448 & 16,4 & 565 & 25,7 & 1,24 & $1,14-1,36^{*}$ \\
\hline Trabalho passivo & 893 & 32,6 & 688 & 31,3 & 0,97 & $0,89-1,06$ \\
\hline Baixo desgaste & 800 & 29,2 & 460 & 20,9 & 0,81 & $0,74-0,90^{*}$ \\
\hline \multicolumn{7}{|l|}{ Suporte social } \\
\hline Alto suporte social & 1.227 & 47,7 & 1.014 & 49,8 & 1 & \\
\hline Baixo suporte social & 1.346 & 52,3 & 1.024 & 50,2 & 0,72 & $0,67-76^{\star}$ \\
\hline \multicolumn{7}{|l|}{ Como você avalia sua qualidade de vida } \\
\hline Boa & 2.256 & 80,9 & 1.516 & 67,3 & 1 & \\
\hline Ruim & 533 & 19,1 & 735 & 32,7 & 1,44 & $1,36-1,53^{*}$ \\
\hline \multicolumn{7}{|l|}{$\begin{array}{l}\text { Quão satisfeito você está com sua } \\
\text { capacidade de trabalho }\end{array}$} \\
\hline Satisfeito & 2.001 & 72,1 & 1.343 & 60,0 & 1 & \\
\hline Insatisfeito & 774 & 27,9 & 894 & 40,0 & 1,33 & $1,26-1,42^{\star}$ \\
\hline \multicolumn{7}{|l|}{ Demanda física no trabalho } \\
\hline Baixa demanda física & 1.613 & 57,1 & 1.010 & 44,1 & 1 & \\
\hline Alta demanda física & 1.211 & 42,9 & 1.278 & 55,9 & 1,33 & $1,25-1,42^{*}$ \\
\hline \multicolumn{7}{|l|}{ Condições de trabalho } \\
\hline Ótimas & 518 & 19,8 & 247 & 11,4 & 1 & \\
\hline Satisfatórias & 862 & 33,0 & 582 & 26,9 & 1,25 & $1,11-1,41^{\star}$ \\
\hline Razoáveis & 567 & 21,7 & 570 & 26,4 & 1,55 & $1,38--1,75^{\star}$ \\
\hline Precárias & 664 & 25,4 & 762 & 35,3 & 1,65 & $1,48-1,85^{\star}$ \\
\hline
\end{tabular}

Fonte: Diagnóstico de Saúde do Servidor e do Empregado Público da Prefeitura Municipal de Belo Horizonte.

${ }^{*} \mathrm{p}<0,001$.

A Tabela 5 apresenta os resultados do modelo final da análise multivariada $(p \leq 0,05)$. Relatos de absenteísmo-doença foram significativamente maiores entre as mulheres (RP 1,12) e a prevalência de absenteísmo diminuiu com o aumento da faixa etária: 30-39 anos (RP 0,079); 40-49 anos (RP 0,072); e 50 anos ou mais (RP 0,068). Quanto às categorias ocupacionais, o absenteísmo mostrou-se associado aos grupos de fiscalização e vigilância (RP 1,46), serviços gerais (RP 1,22), informação, educação e cultura (RP 1,50) e saúde (RP 1,50), quando comparados ao grupo administrativo, contábil financeiro, jurídico e planejamento. 


\section{TABELA 5}

Estatísticas do ajuste do modelo de Poisson multivariado com variáveis estatisticamente significantes associadas ao absenteísmo-doença para servidores e empregados públicos municipais das administrações direta e indireta que responderam ao questionário da pesquisa Município de Belo Horizonte - 2009

\begin{tabular}{|c|c|c|}
\hline Variáveis & Razão de prevalência & IC (95\%) \\
\hline \multicolumn{3}{|l|}{ Sexo } \\
\hline Homens & 1 & \\
\hline Mulheres & 1,12 & $1,03-1,22^{\star \star}$ \\
\hline \multicolumn{3}{|l|}{ Idade } \\
\hline Até 29 anos & 1 & \\
\hline De 30 a 39 anos & 0,79 & $0,72-0,87^{\star \star \star}$ \\
\hline De 40 a 49 anos & 0,72 & $0,65-0,79^{\star \star *}$ \\
\hline 50 anos ou mais & 0,68 & $0,61-0,76^{\star \star \star}$ \\
\hline \multicolumn{3}{|l|}{ Grupos ocupacionais } \\
\hline Administrativo, contábil, financeiro, jurídico e planejamento & 1 & \\
\hline Fiscalização e vigilância & 1,46 & $1,24-1,71^{\star \star \star}$ \\
\hline Serviços gerais & 1,22 & $1,03-1,45^{\star}$ \\
\hline Construção e exploração de recursos naturais & 1,09 & $0,83-1,45$ \\
\hline Informação, educação e cultura & 1,50 & $1,37-1,66^{\star \star \star}$ \\
\hline Saúde & 1,50 & $1,37-1,66^{\star \star \star}$ \\
\hline \multicolumn{3}{|l|}{ Possui diagnóstico médico de LER/Dort } \\
\hline Não & 1 & \\
\hline $\operatorname{Sim}$ & 1,19 & $1,09-1,30^{\star \star \star}$ \\
\hline \multicolumn{3}{|l|}{ Possui diagnóstico médico de lombalgia } \\
\hline Não & 1 & \\
\hline Sim & 1,16 & $1,08-1,25^{\star \star \star}$ \\
\hline \multicolumn{3}{|l|}{ Possui diagnóstico médico de depressão/ansiedade } \\
\hline Não & 1 & \\
\hline Sim & 1,20 & $1,09-1,31^{\star \star \star}$ \\
\hline \multicolumn{3}{|l|}{ Percepção de cansaço mental } \\
\hline Não & 1 & \\
\hline $\operatorname{Sim}$ & 1,12 & $1,04-1,21^{\star \star}$ \\
\hline \multicolumn{3}{|l|}{ Usa medicamento para depressão/ansiedade } \\
\hline Não & 1 & \\
\hline Sim & 0,82 & $0,75-0,89^{\star * *}$ \\
\hline \multicolumn{3}{|l|}{ Transtorno mental comum (SRQ20) } \\
\hline Menor probabilidade de transtorno mental & 1 & \\
\hline Maior probabilidade de transtorno mental & 1,23 & $1,13-1,33^{\star \star \star}$ \\
\hline \multicolumn{3}{|l|}{ Quão satisfeito você está com sua capacidade de trabalho } \\
\hline Satisfeito & 1 & \\
\hline Insatisfeito & 1,08 & $1,01-1,16^{\star}$ \\
\hline \multicolumn{3}{|l|}{ Condições de trabalho } \\
\hline Ótimas & 1 & \\
\hline Satisfatórias & 1,05 & $0,93-1,19$ \\
\hline Razoáveis & 1,21 & $1,07-1,36^{\star \star}$ \\
\hline Precárias & 1,19 & $1,06-1,34^{\star \star}$ \\
\hline
\end{tabular}

Fonte: Diagnóstico de Saúde do Servidor e do Empregado Público da Prefeitura Municipal de Belo Horizonte.

${ }^{\star} p<0,05 ;{ }^{* *} p<0,01 ;{ }^{* \star} p<0,001$ 
Relatos de diagnóstico médico de LER/Dort (RP 1,19), lombalgia (RP 1,16), depressão/ ansiedade (RP 1,20) e percepção de cansaço mental (RP 1,12) foram associados ao absenteísmo-doença quando comparados àqueles que negaram diagnóstico médico confirmado para tais enfermidades. $\mathrm{O}$ uso de medicamento para depressão/ansiedade (RP 0,82 ) se mostrou fator protetor (Tabela 5).

Maior probabilidade de transtorno mental comum (RP 1,23), insatisfação com a capacidade de trabalho (RP 1,08) e condições razoáveis (RP 1,21) e precárias de trabalho (RP 1,19) foram associadas ao absenteísmo-doença (Tabela 5).

\section{Discussão}

Este estudo analisou o perfil de 5.646 servidores públicos municipais que relataram, por ocasião de um inquérito, absenteísmo-doença nos últimos 12 meses. Confirmando os resultados da literatura (BARBOZA; SOLER, 2003; ERIKSSON et al., 2008; HOOFTMAN et al., 2008), foram observadas proporção significantemente maior de absenteísmo-doença entre as mulheres e diminuição com o aumento da idade.

As evidências indicam que o padrão do absenteísmo (frequentes licenças de curta duração ou raras licenças de longa duração) difere entre os sexos (ERIKSSON et al., 2008; HOOFTMAN et al., 2008) devido a questões de gênero. As mulheres são mais vulneráveis às circunstâncias familiares que interferem no trabalho, explicando, em certa medida, maior prevalência de absenteísmo-doença quando comparadas aos homens (ROELEN et al., 2010).

No estudo de Barboza e Soler (2003), a taxa de absenteísmo-doença foi maior entre as trabalhadoras de enfermagem quando comparadas aos seus colegas do sexo masculino. No grupo dos servidores em Santa Catarina, o absenteísmo-doença (com até 15 dias de afastamento) também foi mais frequente (75,90\%) entre as mulheres (CUNHA et al., 2009). Roelen et al. (2010) evidenciaram que a duração do absenteísmo não foi diferente entre homens e mulheres quando calculadas as médias, no entanto, absenteísmo de longa duração (superior a oito semanas) foi mais frequente entre as mulheres.

Encontrou-se proporção significantemente maior de mulheres com pelo menos um episódio de licença (18,2\% entre as mulheres e 10,5\% entre os homens) no estudo que avaliou 21.500 licenças médicas segundo os registros de 58.196 funcionários públicos. Mas a análise por regressão logística mostrou que os homens tendem a tirar licenças de maior duração, com mais frequência, independentemente da idade, do local de trabalho ou do grupo de função exercida. Os autores sugerem que homens possam estar se afastando por doenças de maior gravidade ou de maior impacto sobre a capacidade para o trabalho (SALA et al., 2009).

Coerente aos resultados de Eriksson et al. (2008), observou-se que jovens se afastaram mais frequentemente do que os servidores mais velhos. Contrariamente, Silva et al. (2008) relatam a predominância de absenteísmo-doença entre os homens e em faixas etárias mais elevadas de uma população de bancários. Os autores aventam a hipótese de uma 
sobreposição de efeitos - as mudanças esperadas com o avançar da idade e o maior tempo de exposição aos riscos ocupacionais em trabalhadores menos jovens - para explicar os resultados encontrados. No grupo de servidores estudados por Cunha et al. (2009), o evento foi predominante na faixa de 41 a 50 anos de idade. Por sua vez, Sala et al. (2009) descrevem tendência significante de aumento na chance de ocorrência de licença médica somente a partir dos 60 anos de idade.

Os resultados do presente estudo indicam associação positiva entre absenteísmo-doença e condições inadequadas de trabalho e também com a insatisfação quanto à capacidade para o trabalho. A autoavaliação da capacidade para o trabalho e a decisão de se ausentar do trabalho não são determinadas apenas pela gravidade do problema de saúde, mas também por uma série de fatores sociais, psicológicos e físicos. Já as condições de trabalho podem não apenas contribuir para a doença, mas também influenciar a percepção da capacidade de trabalho (ERIKSEN et al., 2003), podendo explicar, ao menos em parte, os resultados encontrados.

Em convergência aos dados de literatura, a ocorrência de morbidades como LER/Dort e lombalgia, depressão-ansiedade e transtornos mentais comuns (TMC) aumentou a prevalência de absenteísmo-doença (SILVA; MARZIALE, 2003; DUIJTS et al., 2007; ERIKSSON et al., 2008). Cansaço mental permaneceu associado após o ajuste do modelo. Tal resultado é coerente ao achado que confirma associação entre TMC e absenteísmo.

A carga da doença e a prevalência de TMC são elevadas mundialmente com prejuízos para os indivíduos e sociedade (STANSFELD et al., 2011). Na União Europeia, os TMC estão entre os principais fatores relacionados ao absenteísmo, principalmente os casos de depressão, ansiedade e distúrbios associados ao estresse. Em uma coorte de 9.904 trabalhadores na Dinamarca, observou-se risco aumentado de recorrência de absenteísmo no grupo de empregados com episódios prévios de absenteísmo devido aos TMC (KOOPMANS et al., 2011).

Quanto às morbidades musculoesqueléticas, o absenteísmo decorrente de LER/Dort e lombalgia pode ser atribuído às limitações que a dor de origem musculoesquelética provoca, mas também à menor capacidade física devido às deficiências relacionadas às degenerações musculoesqueléticas esperadas em vigência de tais morbidades. No estudo de Silva et al. (2008), também se destacaram as doenças do sistema musculoesquelético seguidas dos transtornos mentais, respondendo por 55,7\% e 19,28\% dos afastamentos, respectivamente. No trabalho de Andréa et al. (2003), a presença de pelo menos uma doença crônica foi preditora relevante para o absenteísmo-doença de longa duração.

Em suma, pode ser que padrões de absenteísmo estejam coincidindo com os períodos de exacerbação de doenças crônicas. Não seria imprudente afirmar que repetidos episódios de absenteísmo-doença constituam estratégias de enfrentamento aos episódios de dor (ROELEN et al., 2011), comuns nos casos de confirmação de diagnóstico de lombalgia e de LER/Dort.

Está identificada uma forte e consistente associação negativa entre a satisfação com as condições psicossociais de trabalho e o absenteísmo (RAEL et al., 1995; KIVMAKI et al., 2002; ANDRÉA et al., 2003; RUGULIES et al., 2007; RAUHALA et al., 2007). Contrariamente 
ao relatado na literatura, no presente estudo, os fatores psicossociais de trabalho não foram associados ao absenteísmo-doença. Entre eles, destaca-se a ausência de associação entre trabalho passivo e o desfecho anunciada na literatura específica (NORTH et al., 1996; MUNCHHANSEN et al., 2009). Tais resultados talvez sejam explicados pelo método empregado na análise, pois o estudo de 1.307 enfermeiras de hospitais públicos concluiu que os instrumentos para avaliar os fatores psicossociais do trabalho foram melhores preditores para o absenteísmo quando usados separadamente (GRIEP, 2010).

Vale mencionar que algumas discrepâncias entre os estudos podem ser atribuídas aos fatores subjacentes à decisão de faltar ou não ao trabalho por um motivo de doença. A decisão em comunicar um adoecimento é uma ação tomada em determinado quadro cultural e social, cujas características não são facilmente traduzidas em variáveis observáveis. Sabe-se que a percepção quanto ao apoio da gestão do trabalho está associada ao absenteísmo-doença (ERIKSEN et al., 2003). Sob esse prisma, é possível que as categorizações para as análises não tenha sido suficientemente potentes para discriminar tais dimensões implicadas na decisão de procurar a assistência médica diante de um problema de saúde.

Como afirmado anteriormente, a relação entre absenteísmo e doença não é direta, uma vez que, em muitos casos, como já citado, o trabalhador doente não necessariamente se ausenta do trabalho. O processo de adoecer, faltar ao trabalho, melhorar e retornar ao trabalho pode indicar falta de equilíbrio entre a pessoa e o ambiente. Dito de outra forma, os problemas de saúde podem surgir em resultado de uma discrepância entre a carga de trabalho (exigências e necessidades) e a capacidade do trabalhador (aptidões e competências), mas sofrem influências organizacionais que interferem na motivação para não faltar e na pressão para comparecer, que se referem à oportunidade e à necessidade do absenteísmo (ROELEN et al., 2010).

Por fim, sabe-se que a duração do afastamento por doença até o retorno ao trabalho é relacionada diretamente ao tempo requerido para a recuperação (ROELEN et al., 2010). Contudo, é também certo tratar-se de um processo dinâmico que envolve não somente o restabelecimento da saúde físico-mental, mas também inclui a adaptação ao trabalho e capacidade de resposta às demandas de desempenho.

Assumindo a complexidade do fenômeno estudado, a presente investigação abordou fatores das diferentes dimensões identificadas pelos autores que trouxeram aclarações sobre as implicações das condições objetivas e subjetivas nas causas do absenteísmo e nos comportamentos dos indivíduos atingidos, considerando a inserção do respondente em seu ambiente de vida e de trabalho.

A força deste estudo é a amplitude das características abarcadas, que compreendem desde fatores individuais relacionados ao trabalho (inserção na ocupação e percepção da exposição a condições físicas e psicossociais dos ambientes de trabalho), até fatores individuais que extrapolam o outro âmbito (estado civil, número de filhos, por exemplo).

Se, por um lado, a heterogeneidade dos indivíduos da amostra quanto às características ocupacionais e sociodemográficas viabilizou a exploração dos múltiplos aspectos 
mencionados, por outro, é possível que variáveis latentes, comuns em populações heterogêneas, tenham explicado ausências ou discrepâncias nos achados quando comparados aos de outros estudos. Remarca-se o caráter homogêneo quanto ao padrão de emprego estável, permitindo descartar hipóteses explicativas quanto aos efeitos das condições precárias de vida que o emprego instável ou o desemprego costumam determinar (BENACH; MUNTANER, 2007).

No Brasil, são raros os estudos que abordam autorrelatos de populações ocupadas, ativas e empregadas sob um mesmo regime, mas diferenciadas quanto aos demais aspectos sociodemográficos e às características ocupacionais (condições físicas e psicossociais de trabalho, por exemplo). Em sua maioria, os autores utilizam dados registrados nos serviços de medicina e segurança do trabalho (SILVA; MARZIALE, 2003; REIS et al., 2003; BARBOZA; SOLER, 2003; SILVA et al., 2008; CUNHA et al., 2009). Tais estudos sobre absenteísmo têm focalizado grupos formalmente afastados do trabalho com justificativas baseadas em declarações médicas. Em Belo Horizonte, foram concedidas, em 2000, 1.364 licenças médicas aos servidores universitários, média de 2,15 e máximo de 12 por trabalhador atendido no serviço de medicina ocupacional. Em torno de 36\% dos trabalhadores registraram mais de um afastamento (REIS et al., 2003).

Constatou-se que 59,1\% dos trabalhadores da enfermagem se afastaram pelo menos uma vez no ano considerado. Destacaram-se os casos de um enfermeiro que se afastou 17 vezes e de um auxiliar de enfermagem com registro de 22 episódios de afastamentos do trabalho (BARBOZA; SOLER, 2003).

A variável modelo demanda e controle foi baseada na combinação das dimensões demanda psicológica e controle sobre o trabalho, obtidas por meio do Demand, Control and Support Questionnaire (DCSQ), versão brasileira reduzida (17 questões) do questionário sueco DCSQ. Tal combinação resultou em quatro categorias: trabalho ativo (alta demanda, alto controle); baixo desgaste (baixa demanda, alto controle); alto desgaste (alta demanda, baixo controle); e trabalho passivo (baixa demanda, baixo controle). O questionário refere-se às fontes de estresse no ambiente psicossocial do trabalho e ao desgaste resultante de sua interação (GRIEP et al., 2011).

As limitações do presente estudo devem ser discutidas. A amostra não probabilística incluiu servidores apenas da Prefeitura de Belo Horizonte, de forma que comparações com outras populações merecem cautela. A taxa de resposta do inquérito foi de $14 \%$, apesar das estratégias para estimular a adesão dos elegíveis e diminuir as disparidades no acesso ao veículo da pesquisa (foram disponibilizados laptops em áreas onde não havia tais equipamentos). É provável que os resultados obtidos tenham sido subestimados, considerando-se a maior concentração de respondentes de alta escolaridade que está atrelada a melhor condição de saúde.

A baixa taxa de resposta, a hiper-representação dos mais escolarizados e a inelegibilidade daqueles que não estavam presentes ou que não participaram por abandono, exoneração ou afastamento por doença podem ter influenciado as associações encontradas. 
Comparados aos servidores do setor administrativo, sujeitos de todas as ocupações, com exceção da construção e exploração de recursos naturais, apresentaram maior prevalência de absenteísmo-doença. Esse resultado não permitiu aprofundamentos porque a categorização mereceria distinções mais destacadas entre os subgrupos populacionais. Quanto a estes, foram tipicamente classificados de acordo com sexo, idade, características pessoais, comportamentos, ocupação. Mas ainda permanecem obscuras as dimensões das condições psicossociais do trabalho que estariam associadas de maneiras distintas ao absenteísmodoença a depender dos subgrupos ocupacionais que não foram operacionalizados no delineamento da pesquisa. Vale lembrar que características locais da cultura organizacional podem ser mais relevantes do que as categorias ocupacionais classificadas em grandes grupos para explicar o absenteísmo-doença (MUNCH-HANSEN et al., 2008).

Apesar dos limites mencionados, o presente estudo ganha em relevância, tendo em vista o número de participantes (superior a 5.000 servidores), a incorporação de diferentes ocupações e a variedade de medidas referentes às características dos indivíduos e do trabalho. Estas medidas são necessárias para identificar vulnerabilidades ocupacionais e podem contribuir para o desenvolvimento de políticas de ação pró-equidade nesse universo populacional, bem como um acompanhamento longitudinal das ações propostas. A estabilidade de emprego da população foco abre possibilidade de monitoramento permanente de possíveis disparidades e avaliação de programas sob a ótica da equidade, essenciais para que estes trabalhadores alcancem e conservem seu potencial de saúde. Outra vantagem foi trazer resultados baseados em informações colhidas diretamente dos trabalhadores, ampliando o conhecimento sobre os cotidianos de trabalho tal como eles são vivenciados e representados pelos sujeitos.

As pesquisas realizadas via web têm sido encaradas como mais vantajosas do que aquelas empreendidas por meio dos veículos tradicionais para a coleta de dados: custo reduzido; facilidade e rapidez na administração; maior garantia de anonimato; alcance em larga escala; e tempo curto para resposta. Além disso, a natureza e a qualidade das respostas não são afetadas. Alguns autores sugerem que as pesquisas on-line são apropriadas por alcançarem respostas completas e por não se configurarem como respostas apresentadas para "agradar" o entrevistador (GANASSALI, 2008; EVANS; MATHUR, 2005). Não obstante, vários riscos são possíveis: a ausência de interação com o entrevistador impede verificações para assegurar a fiabilidade; não é possível controlar as amostras, nem identificar a sua representatividade por causa da garantia do anonimato (FOX et al., 2003). Devido a esse último motivo, não foi controlado o local ou o computador de onde o questionário foi acessado.

\section{Conclusão}

Relatos de absenteísmo-doença foram significativamente maiores entre as mulheres e a prevalência de absenteísmo diminuiu com o aumento da faixa etária. Morbidades musculoesqueléticas diagnosticadas (LER/Dort e lombalgias), de um lado, e depressão/ 
ansiedade, percepção de cansaço mental e transtorno mental comum, de outro lado, também foram significativamente associadas ao absenteísmo-doença. Insatisfação com a capacidade de trabalho e condições inadequadas de trabalho também se mostraram significativamente associadas ao desfecho.

\section{Referências}

ALVES, M. G. M.; CHOR, D.; FAERSTEIN, E.; LOPES, C. S.; WERNECK, G. L. Versão resumida da "job stress scale" adaptação para o português. Revista de Saúde Pública, v. 38, n. 2, p. 164-71, 2004.

ALVES, M.; CERVINHO, S.; GODOY, B. Absenteísmo-doença entre funcionários de um hospital universitário. Revista Brasileira de Saúde Ocupacional, v. 103, n. 27, p. 33-48, 2002.

ANDREA, H.; BEURSKENS, A. J.; METSEMAKERS, J. F.; VAN AMELSVOORT, L. G. P. M.; VAN DEN BRANDT, P. A.; VAN SCHAYCK, C. P. Health problems and psychosocial work environment as predictors of long term sickness absence in employees who visited the occupational physician and/or general practitioner in relation to work: a prospective study. Occupational Environmental Medicine, v. 60, n. 4, p. 295-300, 2003.

BARROS, A. J. D.; HIRAKATA, V. N. Alternatives for logistic regression in cross-sectional studies: an empirical comparison of models that directly estimate the prevalence ratio. BMC Medical Research Methodology, v. 3, n. 21, 2003.

COUTINHO, L. M. S.; SCAZUFCA, M.; MENEZES, P. R. Métodos para estimar razão de prevalência em estudos de corte transversal. Revista de Saúde Pública, v. 42, n. 6, p. 992-998, 2008.

BARBOZA, D. B.; SOLER, Z. A. S. G. Afastamentos do trabalho na enfermagem: ocorrências com trabalhadores de um hospital de ensino. Revista Latino-Americana de Enfermagem, v. 11, n. 2, p. 177-83, 2003.

BENACH, J.; MUNTANER, C. Precarious employment and health: developing a research agenda. Journal Epidemiology Community Health, v. 7. n. 61, p. 276-7, 2007.

BOWERS, T. Teacher absenteeism and ill health retirement: a review. Cambridge Journal Education, v. 31, n. 2, p.135-157, 2001.

CAIL, F.; PICHENE, A.; APTEL, M. Questionnaire d'évaluation du vécu du travail desalariés exposés à des risques de troubles musculosquelettiques. Documents Pour le Medecin du Travail, v. 12, n. 64, p. 253-267, 1995.

CHIAVENATO, I. Planejamento de recursos humanos. Recursos humanos na empresa. São Paulo: Atlas, 1981, p. 27-52.

CUNHA, J. B.; BLANK, V. L. G.; BOING, A. F. Tendência temporal de afastamento do trabalho em servidores públicos (1995-2005). Revista Brasileira de Epidemiologia, v. 12, n. 2, p. 226-236, 2009.

DUIJTS, S. F. A.; KANT, I.; SWAEN, G. M. H.; VAN DEN BRANDT, P. A.; ZEEGERS, M. P. A. A meta-analysis of observational studies identifies predictors of sickness absence. Journal of Clinical Epidemiology, v. 60, p. 1105-1115, 2007.

EHRENBEG, R. G.; EHRENBERG, R. A.; REES, D. I.; EHRENBERG, E. L. School district leave policies, teacher absenteeism, and student achievement. Cambridge: National Bureau of Economic Research, 1989 (Working paper, n. 2874).

ERIKSEN, W.; BRUUSGAARD, D.; KNARDAHL, S. Work factors as predictors of sickness absence: a three month prospective study of nurses' aides. Occupational Environmental Medicine, v. 60, p. 271-278, 2003.

ERIKSSON, H.-G.; VON CELSING, A.-S.; WAHLSTRÖM, R.; JANSON, L.; ZANDER, V.; WALLMAN, T. SicknesS absence and self-reported health a population-based study of 43,600 individuals in central Sweden.

BMC Public Health, v. 8, n. 426, 2008. 
EUROPEAN FOUNDATION FOR THE IMPROVEMENT OF LIVING AND WORKING CONDITIONS (EFILWC). Preventing absenteeism at the workplace - European Research Report. Luxembourg: Office for Official Publications of the European Communities, 1997.

EVANS, J. R.; MATHUR, A. The value of online surveys. Internet Research, v. 15, p. 195-219 [online], 2005.

FOX, J.; MURRAY, C.; WARM, A. Conducting research using web-based questionnaires: practical, methodological, and ethical considerations. International Journal of Social Research Methodology, v. 6, p. 167-180, 2003.

GANASSALI, S. The influence of the design of web survey questionnaires on the quality of responses. Survey Research Methods, v. 2, p. 21-32, 2008.

GRIEP, R. H.; ROTENBERG, L.; LANDSBERGIS, P. et al. Uso combinado de modelos de estresse no trabalho e a saúde auto-referida na enfermagem. Revista de Saúde Pública, v. 45, n. 1, p. 1-8, 2011.

GRIEP, R. H.; ROTENBERG, L.; CHOR, D.; LANDSBERGIS, P. Beyond simple approaches to studying the association between work characteristics and absenteeism: Combining DCS and the ERI models. Work and Stress, v. 24, p. 179-195, 2010.

HOOFTMAN, W. E.; WESTERMAN, M. J.; VAN DER BEEK, A. J.; BONGERS, P. M.; VAN MECHELEN, W. What makes men and women with musculoskeletal complaints decide they are too sick to work? Scandinavian Journal of Work, Environment \& Health, v. 34, n. 2, p. 107-112, 2008.

KARASEK, R. A. Job demands, job decision latitude, and mental strain: implications for job redesign. Administrative Science Quarterly, v. 24, n. 2, p. 285-307, 1979.

KIVIMAKI, M.; VAHTERA, J.; ELOVAINIO, M.; LILLRANK, B.; KEVIN, M. V. Death or illness of a family member, violence, interpersonal conflict, and financial difficulties as predictors of sickness absence: longitudinal cohort study on psychological and behavioral links. Psychosomatic Medicine, v. 64, p. 817-825, 2002.

KOOPMANS, P. C.; BÜLTMANN, U.; ROELEN, C. A. M.; HOEDEMAN, R.; VAN DER KLINK, J. J. L.; GROOTHOFF, J. W. Recurrence of sickness absence due to common mental disorders. International Archives Occupational Environmental Health, v. 84, p. 193-201, 2011.

MUNCH-HANSEN, T.; WIECLAW, J.; AGERBO, E.; WESTERGAARD-NIELSE, N.; BONDE, J. P. Global measure of satisfaction with psychosocial work conditions versus measures of specific aspects of psychosocial work conditions in explaining sickness absence. BMC Public Health, v. 8, n. 270, 2008.

Sickness absence and workplace levels of satisfaction with psychosocial work conditions at public service workplaces. American Journal of Industrial Medicine, v. 52, p. 153-161, 2009.

NORTH, F. M.; SYME, S. L.; FEENEY, A.; SHIPLEY, M.; MARMOT, M. Psychosocial work environment and sickness absence among british civil servants: the Whitehall II study. American Journal of Public Health, v. 86, n. 3, p. 332-340, 1996.

OFICINA INTERNACIONAL DEL TRABAJO (OIT). Enciclopedia de salud, seguridad e higiene en el trabajo. Madrid: Centro de Publicaciones del Ministerio de Trabajo y Seguridad Social, 1991.

RAEL, E. G.; STANSFELD, S. A.; SHIPLEY, M.; HEAD, J.; FEENEY, A.; MARMOT, M. Sickness absence in the Whitehall II study, London: the role of social support and material problems. Journal of Epidemiology and Community Health, v. 49, p. 474-481, 1995.

RAUHALA, A.; KIVIMAKI, M.; FAGERSTROM, L.; ELOVAINIO, M.; VIRTANEN, M.; VAHTERA, J.; RAINIO, A.-K.; OJANIEMI, K.; KINNUNEN, J. What degree of work overload is likely to cause increased sickness absenteeism among nurses? Evidence from de RAFAELA patient classification system. Journal of Advanced Nursing, v. 57, n. 3, p. 286-295, 2007.

REIS, J. R.; LA ROCCA, P. F.; SILVEIRA, A. M.; BONILLA, I. M. L.; GINÉ, N. A.; MARTÍN, M. Fatores relacionados ao absenteísmo por doença em profissionais de enfermagem. Revista de Saúde Pública, v. 37, n. 5 , p. 616-23, 2003. 
ROELEN, C. A. M.; KOOPMANS, P. C.; ANEMA, J. R.; VAN DER BEEK, A. J. Recurrence of medically certified sickness absence according to diagnosis: a sickness absence register study. Journal of Occupational Rehabilitation, v. 20, n. 1, p. 113-121, 2010.

ROELEN, C. A. M.; KOOPMANS, P. C.; SCHREUDER, J. A. H.; ANEMA, J. R.; VAN DER BEEK, A. J. The history of registered sickness absence predicts future sickness absence. Occupational Medicine, v. 61, n. 2, p. 96-101, 2011.

RUGULIES, R.; CHRISTENSEN, K. B.; BORRITZ, M.; VILLADSEN, E.; BULTMANN, U.; KRISTENSEN, T. S. The contribution of the psychosocial work environment to sickness absence in human service workers: Results of a 3-year follow-up study. Work \& Stress, v. 21, n. 4, p. 293-311, 2007.

SALA, A.; CARRO, A. R. L.; CORREA, A. N.; SEIXAS, P. H. A. Licenças médicas entre trabalhadores da Secretaria de Estado da Saúde de São Paulo no ano de 2004. Cadernos de Saúde Pública, v. 25, n. 10, p. 2168-2178, 2009.

SANTOS, K. O. B.; ARAÚJO, T. M.; OLIVEIRA, N. F. Estrutura fatorial e consistência interna do Self-Reporting Questionnaire (SRQ-20) em população urbana. Cadernos de Saúde Pública, v. 25, p. 214-222, 2009.

SILVA, D. M. M. P.; MARZIALE, M. H. P. Problemas de saúde responsáveis pelo absenteísmo de trabalhadores de enfermagem de um hospital universitário. Acta Scientiarum. Health Sciences, v. 25, n. 2, p. 191-197, 2003.

Absenteísmo de trabalhadores de enfermagem em um hospital universitário. Revista Latino-americana de Enfermagem, v. 8, n. 5, p. 44-51, 2000.

SILVA, L. S.; PINHEIRO, T. M. M.; SAKURAI, E. Perfil do absenteísmo em um banco estatal em Minas Gerais: análise no período de 1998 a 2003. Ciência \& Saúde Coletiva, v. 13 (Sup. 2), p. 2049-2058, 2008.

STANSFELD S. A.; FUHRER, R.; HEAD, J. Impact of common mental disorders on sickness absence in an occupational cohort study. Occupational Environmental Medicine, v. 68, p. 408-413, 2011.

THEORELL, T.; KARASEK, R. A. Current issues relating to psychosocial job strain and cardiovascular disease research. Journal of Occupational Health Psychology, v. 1, p. 9-26, 1996.

\section{Autores}

Celeste de Souza Rodrigues é doutora em Saúde Pública, médica na Prefeitura de Belo Horizonte.

Rosiene Maria de Freitas é mestre em Saúde Pública, doutoranda do Programa de Pós-Graduação em Ciência Política da Universidade Federal de Minas Gerais - UFMG.

Ada Ávila Assunção é pós-doutora em Saúde Pública, professora do Programa de Pós-Graduação em Saúde Pública da Universidade Federal de Minas Gerais - UFMG.

lara Barreto Bassi é mestre em Saúde Pública, doutoranda do Programa de Pós-Graduação em Saúde Pública da Universidade Federal de Minas Gerais - UFMG.

Adriane Mesquita de Medeiros é doutora em Saúde Pública, professora do Departamento de Fonoaudiologia da Universidade Federal de Minas Gerais - UFMG.

\section{Abstract \\ Absenteeism and sickness as per self-reports by municipal public employees in Belo Horizonte, Brazil}

The purpose of this study was to analyze the profile of municipal public servants who reported illnessrelated absenteeism in the preceding 12 months. The study involved a sample of 5,646 (14\%) of all 38,304 municipal workers in the city of Belo Horizonte, Minas Gerais, Brazil, in 2009. A questionnaire was placed on the Internet, where entry depended on the respondents' consent. Poisson regression 
analysis was used to determine the associated factors. Absenteeism due to illness was highly correlated with women (PR 1.12) and decreased with age: 30-39 years (PR 0.79), 40-49 years (PR 0.72) and 50 years of over (PR 0.68). Sickness-related absenteeism was closely associated with work involving monitoring and surveillance groups (PR 1.46), general services (PR 1.22), information, education and culture (PR 1.50), and health (PR 1.50). Comorbidities included WRMD (PR 1.19), lower-back pain (PR 1.16), depression / anxiety (PR 1.20) and perception of mental fatigue (PR 1.12). The reported use of medication for depression / anxiety proved to be a protective factor (PR 0.82). Factors such as Greater likelihood of mental disorder (PR 1.23) according to SRQ20, Dissatisfaction with one's ability to work (PR 1.08), Reasonable conditions (PR 1.21) and Poor working conditions (PR 1.19), were associated with the outcomes. Health promotion programs would be useful to decrease illness-related absenteeism because interventions for preventing musculoskeletal comorbidity and psychosocial complaints would attenuate situations that are probably the source of absenteeism due to illness.

Keywords: Absenteeism. Civil servants. Sickness. Working conditions. Psychosocial factors.

\section{Resumen}

\section{Ausentismo-enfermedad según auto-relato de empleados públicos municipales en Belo Horizonte}

El objetivo del estudio es analizar el perfil de un conjunto de empleados públicos municipales que relataron, en el marco de una investigación, su ausentismo-enfermedad en los últimos 12 meses. En el 2009 se obtuvo una muestra de 5.646 (14\%) del universo de 38.304 empleados municipales de Belo Horizonte. Se puso a disposición en un sitio de Internet un cuestionario, cuya entrada dependía del consentimiento de la persona que lo contestaría. La regresión de Poisson fue utilizada para determinar los factores asociados. La prevalencia del ausentismo-enfermedad fue mayor entre los empleados del sexo femenino (RP 1,12) y se redujo con el aumento de los grupos de edad: $30-39$ años (RP 0,79); 40-49 años (RP 0,72); y 50 años o más (RP 0,68). El ausentismo-enfermedad se mostró asociado a las actividades vinculadas a los grupos de fiscalización y vigilancia (RP 1,46), servicios generales (RP 1,22), información, educación y cultura (RP 1,50) y salud (RP 1,50). Las comorbilidades más frecuentes fueron LER/Dort (RP 1,19), lumbago (RP 1,16), depresión/ansiedad (RP 1,20) y percepción de cansancio mental (RP1,12). El relato de uso de medicación para depresión/ansiedad se mostró un factor protector (RP 0,82). Una mayor probabilidad de trastorno mental (RP 1,23) según el SRQ20, insatisfacción con la capacidad de trabajo (RP 1,08), condiciones razonables (RP 1,21) y condiciones precarias de trabajo (RP 1,19) fueron asociadas al desenlace. Programas de promoción de la salud en el trabajo con miras a prevenir comorbilidades musculoesqueléticas y quejas psicosociales atenuarían las situaciones que probablemente se sitúan en el origen del ausentismo-enfermedad.

Palabras clave: Ausentismo. Empleados públicos. Enfermedad. Condiciones de trabajo. Factores psicosociales.

Recebido para publicação em 01/09/2011 Aceito para publicação em 04/01/2012 